\title{
A multi-class tactile brain-computer interface based on stimulus-induced oscillatory dynamics
}

\author{
Lin Yao, Mei Lin Chen, Xinjun Sheng, Natalie Mrachacz-Kersting, \\ Xiangyang Zhu, Dario Farina, Ning Jiang*
}

\begin{abstract}
We proposed a multi-class tactile brain-computer interface that utilizes stimulus-induced oscillatory dynamics. It was hypothesized that somatosensory attention can modulate tactile induced oscillation changes, which can decode different sensation attention tasks. Subjects performed four tactile attention tasks, prompted by cues presented in random order and while both wrists were simultaneously stimulated: 1) selective sensation on left hand (SS-L), 2) selective sensation on right hand (SS-R), 3) bilateral selective sensation (SS-B), and 4) selective sensation suppressed or idle state (SS-S). The classification accuracy between SS-L and SS-R $(79.9 \pm 8.7 \%)$ was comparable with that of a previous tactile BCI system based on selective sensation. Moreover, the accuracy could be improved to an average of $90.3 \pm 4.9 \%$ by optimal class-pair and frequency-band selection. Three-class discrimination had accuracy of $75.2 \pm 8.3 \%$, with the best discrimination reached for the classes SS-L, SS-R and SS-S. Finally, four classes were classified with accuracy of 59.4 $\pm 7.3 \%$. These results show that the proposed system is a promising new paradigm for multi-class BCI.
\end{abstract}

Index Terms- Tactile BCI, Selective Sensation, Stimulus-induced Oscillatory Dynamics, Somatosensory Attention

\section{INTRODUCTION}

Brain-computer interface (BCI) provides a non-muscular channel for interaction with the external environment [1], [2]. It has been demonstrated that subjects can voluntarily modulate sensorimotor rhythms (SMR) generated from the motor cortex [3] by performing motor imagery (MI) of their limbs (e.g., left or right hand) [4]-[9]. However, there is a latency, usually in the order of seconds, between the MI task and the generation of SMR patterns [10]-[12], making it difficult to develop a highly interactive BCI. Furthermore, SMR-based BCIs suffer from the issue of "BCI Illiteracy" [13], [14], in that a significant portion of users (15-30\% of the general population) cannot successfully use SMR-based BCI systems even after extensive training [15]-[18]. Alternatively, visual P300 or steady-state visual evoked potential (SSVEP) from EEG can also be used for BCI systems [19], [20], but these fast visual BCI requires full engagement of the users' gaze control, which can be challenging and undesirable in real-life application settings. It is suggested that auditory and proprioceptive BCIs are the only remaining channels of communication for many potential BCI users, such as those in the late stage of amyotrophic lateral

Lin Yao, Mei Lin Chen, and Ning Jiang are with Department of Systems Design Engineering, Faculty of Engineering, University of Waterloo, Waterloo, Canada (E-mail: ning.jiang@uwaterloo.ca).

Xinjun Sheng and Xiangyang Zhu are with State Key Lab of Mechanical System and Vibration, Shanghai Jiao Tong University, Shanghai, China.

Natalie Mrachacz-Kersting is with Center for Sensory-Motor Interaction, the Faculty of Medicine, Aalborg University, Aalborg, Denmark.

Dario Farina is with Department of Bioengineering, Imperial College London, London, UK. sclerosis (ALS) [21]-[23]. By requiring the active involvement of the somatosensory system, somatosensory stimulation might also provide a way to avoid the "extinction of thought" [24]. Thus, BCI systems based on tactile stimulation will not only provide a way for communication with the external world [25], [26], but also help to engage the remaining somatosensory system [24].

A tactile BCI provides a complementary approach to increasing BCI diversity by fully exploring the functioning somatosensory system of the BCI user. The first prototype of a tactile BCI was proposed by Mueller-Putz et al [25], and based on steady-state somatosensory evoked potentials (SSSEP) [27]-[29]. This system does not require eye control. SSSEP is a steady-state component of the brain signal, evoked by sustained vibrotactile stimulation within the frequency range of 17 to 35 $\mathrm{Hz}$ [30]. The evoked amplitude of the SSSEP can be modulated by subjective attention [31]. Experiments on five subjects have shown that the classification accuracy for this BCI modality ranged from $64 \%$ to $84 \%$, with an average accuracy of $70.4 \%$. Four of the investigated subjects attained an accuracy below $70 \%$. Another study on SSSEP showed a mean classification accuracy of $58 \%$ for 16 subjects, with 15 out of 16 subjects below the $70 \%$ accuracy level [32]. Subsequently, a tactile P300 system, similar to the visual P300 BCI, based on the oddball paradigm, was proposed [33]. This system achieved a mean accuracy of $72 \%$ in 11 subjects, when selecting between two targets.

Recently, in a series of studies, we proposed a tactile BCI based on oscillatory dynamics from the somatosensory area of the cortex, which we termed selective sensation (SS) tactile BCI [34]-[36]. This approach is based on the fact that event-related desynchronization/synchronization (ERD/ERS) not only has a strong correlation with real or imagined movement but also with the processing of afferent inflow in the human somatosensory system [37], [38]. The somatosensory attention could also modulate the amplitudes of ERD/ERS [39]. The 43 subjects that we have so far investigated attained an accuracy of $79.2 \%$, with only seven with an accuracy below $70 \%$ [36]. Therefore, this tactile BCI modality based on SS substantially outperformed previous tactile BCI systems, making it potentially applicable to a larger number of users.

In this study, we extended the two-class BCI in our previous studies to a multiple-class tactile BCI system. Four mental tasks were randomly presented to the subjects: selective sensation on the left hand (SS-L), selective sensation on the right hand (SS-R), bilateral selective sensation (SS-B), and selective sensation suppressed or idle state (SS-S). As a first step toward a multi-class tactile BCI, we focused on addressing the question whether two-class classification can be improved by optimal 


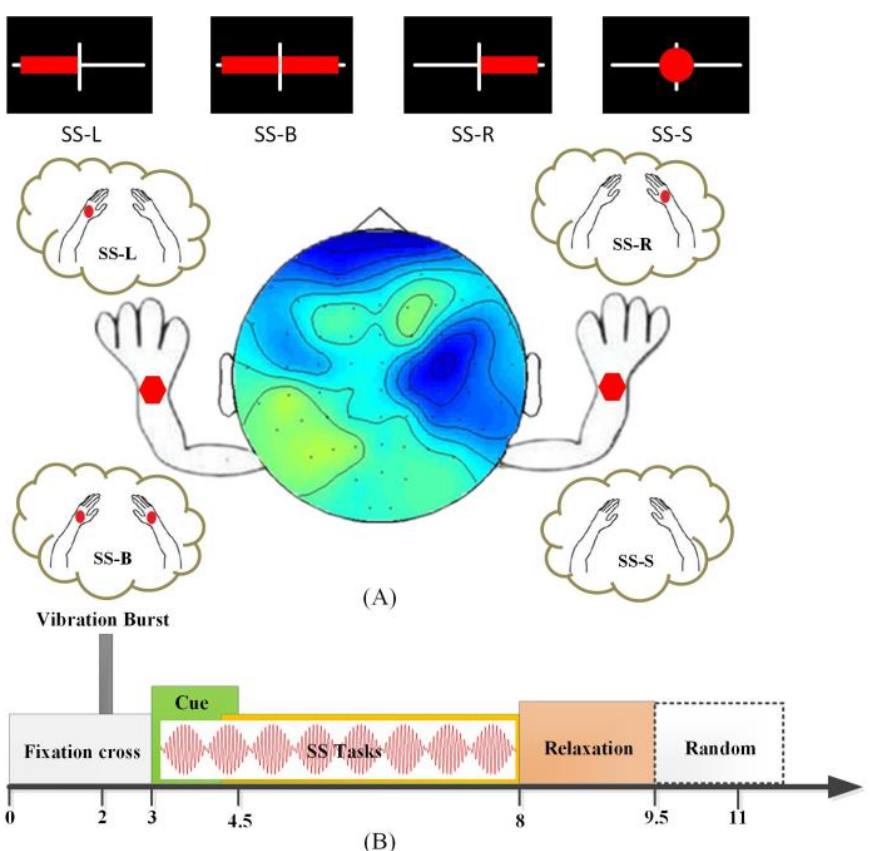

(B)

Figure 1. Illustration of the experiment protocol. (1) Graphic representation of the applied bilateral vibration stimulus (indicated by the two red hexagons), and the four SS tasks (the red dots or the lack of it for SS-S) (2) The temporal sequence of each trial.

selection of pairs of SS tasks. In addition, we tested the performance of three- and four-class discrimination.

\section{METHODOLOGY}

\section{A. Subjects}

Twelve healthy subjects participated in the experiments (five female, seven male, all right handed, average age 21.2 \pm 1.5 years), all were BCI naïve subjects. This study was approved by the Ethics Committee of the University of Waterloo, Waterloo, Canada. All participants signed an informed consent form before participation.

\section{B. EEG Recording and Somatosensory Stimulation}

EEG signals were recorded using a 32-channel wireless g.Nautilus EEG system (g.tec, Austria). The electrodes were placed according to the extended 10/20 system. The reference electrode was located on the right earlobe, and the ground electrode on the forehead. A hardware notch filter at $60 \mathrm{~Hz}$ was applied to the raw signals. The signals were digitally sampled at $250 \mathrm{~Hz}$.

Mechanical stimulation was applied to the wrists. Linear resonant actuators (10 mm, C10-100, Precision Microdrives Ltd., typical normalized amplitude $1.4 \mathrm{G}$ ) were used for producing vibrotactile stimulation. The stimulation device produced a $23-\mathrm{Hz}$ sine wave for the left wrist, and $27-\mathrm{Hz}$ sine wave for the right wrist. Both stimuli were modulated with a $175-\mathrm{Hz}$ sine carrier wave. These stimuli activate the Pacinian and Meissner corpuscles [40], which are sensitive to frequencies above $100 \mathrm{~Hz}$ and $20-50 \mathrm{~Hz}$, respectively. The amplitude of the vibration was individually adjusted to be between the maximum amplitude (11.3 um) and half of the maximum amplitude at the resonant frequency. The selection of the optimal amplitude was based on individual feedback from the subject, such that they were comfortable with perceiving the vibration.

\section{Experimental Protocol}

During the SS task period, sustained vibrotactile stimuli were simultaneously applied to both wrists. Four SS tasks were performed according to different cues: 1 . Selective sensation on the left hand (SS-L); 2. Selective sensation on the right hand (SS-R); 3. Bilateral selective sensation (SS-B); 4. Selective sensation suppressed (SS-S).

The experimental paradigm is illustrated in Fig. 1. The subject was seated on a comfortable armchair, with both forearms and hands resting on the armrests. The subjects were instructed to limit their eye, facial and arm movements. During the experiment, a series of visual cues were presented to the subjects on a computer screen located at a distance of $1 \mathrm{~m}$ from the subjects. At the beginning of each trial, a white fixation symbol ("+") appeared in the center of the screen. After $2 \mathrm{~s}$, a vibration pulse stimulated both hands for $200 \mathrm{~ms}$ with the same intensity, to alert the user of the subsequent task. At the 3rd second, a red cue of the following four types was presented on the computer monitor: 1) a left-pointing arrow corresponding to the SS-L task; 2) a right-pointing arrow corresponding to the SS-R task; 3) a double-sided arrow corresponding to the SS-B task; and 4) a circle corresponding to the SS-S task. This cue was superimposed on the fixation symbol and lasted for $1.5 \mathrm{~s}$. The subjects were instructed to perform the corresponding mental task after the appearance of the cue. The mental task continued for $5 \mathrm{~s}$, until the fixation symbol disappeared. During this period, sustained vibrotactile stimulation was simultaneously applied to both wrists. Next there was a relaxation period lasting $1.5 \mathrm{~s}$. Finally, a random time interval of 0 to $2 \mathrm{~s}$ followed the relaxation period, to prevent subject adaptation. A total of 240 trials (60 trials for each task) were performed by the subjects in 6 runs, each of which consisted of 10 trials of each task in random order. There were 2-4 min breaks between two consecutive runs.

\section{Calculation of ERD/ERS and time frequency decomposition}

Event related desynchronization (ERD) and event related synchronization (ERS) are defined as the percentage of power decrease (ERD) and power increase (ERS) in a defined frequency band in relation to a reference interval (usually taken at a different time interval) [41]. The frequency band alpha-beta of [8 26] Hz was adopted in this study for EEG filtering before the ERD/ERS calculation. The reference interval for the ERD/ERS calculation was from $1.2 \mathrm{~s}$ to $2.0 \mathrm{~s}$ prior to the appearance of the cue. The grand averaged ERD/ERS curves from all subjects of the same task were used to determine the activation and deactivation of the cortex areas involved in the mental tasks.

The EEG data was manually corrected for artifacts using the EEGLAB toolbox [42]. Trials contaminated with swallowing and physical movement artifacts (either in baseline or task interval) were excluded from the analysis. For all subjects, at least 45 trials (over all classes) were used for subsequent 
analysis (while no trials were discarded for the classification evaluation). Time-frequency decomposition of each trial along each EEG channel was performed to construct the spatio-spectral-temporal structure according to the pre-defined mental tasks. It was calculated every $200 \mathrm{~ms}$ with a hanning tapper, convoluted with a modified sinusoid basis, in which the number of cycles linearly changed with frequency to achieve proper time and frequency resolution [43]. The $\mathrm{R}^{2}$ index (squared Pearson-correlation coefficient between feature and class label) [44], [45] was calculated based on the above spatio-spectral-temporal structures between different mental tasks, and used to locate the component of different EEG channels for the classification of the corresponding mental tasks. The Discriminative Brain Pattern (DBP) was defined as a topographic plot of the $\mathrm{R}^{2}$ index, which was averaged along the task time interval mentioned above, and along certain frequency bands, such as alpha $(8-13 \mathrm{~Hz})$, beta $(13-26 \mathrm{~Hz})$, or alpha-beta $(8-26 \mathrm{~Hz})$.

\section{E. Algorithms and Performance Evaluation}

Spatial filtering was adopted to reduce the number of channels and to enhance the feature discrimination among the investigated SS tasks. The spatial filters were determined with the Common Spatial Pattern (CSP) procedure, which has been extensively validated for BCIs [46], [47]. The log-variance of the first and last three components produced by CSP were chosen as feature vectors, and linear discriminative analysis (LDA) was used for classification. As the most discriminative frequency bands are highly subject-dependent, the bands were selected as: lower alpha [8 10] Hz ( $\alpha-)$, upper alpha [10 13] Hz

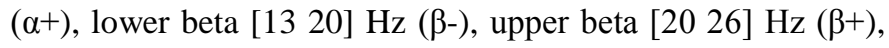
alpha [8 13] Hz $(\alpha)$, beta [13 26] Hz ( $\beta)$, alpha-beta [8 26] Hz $(\alpha \beta)$, and eta $\left[\begin{array}{ll}10 & 16\end{array}\right] \mathrm{Hz}(\eta)$. A fourth-order Butterworth filter was applied to the raw EEG signals before the CSP spatial filtering. All available channels were used in CSP calculation. A $10 \times 10$ fold cross-validation was utilized to evaluate the BCI performance among different frequency bands, and for selecting the optimal frequency band.

EEG signals were segmented from $1 \mathrm{~s}$ to $4 \mathrm{~s}$ after the appearance of the cue (the timing interval of the 4th to 7 th seconds from the beginning of the trial) for the analysis. For the two-class scenario, two out of the four classes were selected for discriminative analysis, resulting in six two-class cases: SS-L vs SS-R (P1), SS-L vs SS-B (P2), SS-L vs SS-S (P3), SS-R vs SS-B (P4), SS-R vs SS-S (P5), and SS-B vs SS-S (P6). Similarly, four three-class cases were investigated: SS-L vs SS-R vs SS-B (T1), SS-L vs SS-R vs SS-S (T2), SS-L vs SS-B vs SS-S (T3), and SS-R vs SS-B vs SS-S (T4). Further, the four-class classification was also performed. To investigate the general applicability of the proposed SS tactile BCI system, the performance for the two-class, three-class and four-class cases was analyzed with fixed frequency bands for all subjects. In addition, as there was a large inter-subject variability, we also performed subject-specific optimization of frequency bands and class pairs, to explore the best possible performance for individual subjects.

\section{F. Statistics}

One-way ANOVA with repeated-measures was used to analyze performance differences among different BCI task pairs (with $p=0.05$ ), and multiple comparisons with Bonferroni correction was used for post-hoc comparison whenever the main effect was found to be significant. For the interpretation of the classification result, the theoretical chance level was corrected with the number of the trials [48]. The corrected chance accuracy for $\mathrm{p}=0.05$ for two-class classification was $61.67 \%$, for three-class $42.78 \%$, and for four-class $32.50 \%$.

\section{RESULTS}

\section{A. Oscillatory Dynamics of Selective Sensation Tasks}

Fig. 2 shows the grand-averaged oscillatory dynamics across four different tactile sensation tasks. At the $-1 \mathrm{~s}$, a vibration burst of $200 \mathrm{~ms}$ was applied to both wrists to alert subjects to get ready for the tasks. This corresponded to a clear simultaneous alpha-beta frequency power reduction for both $\mathrm{C} 3$ and $\mathrm{C} 4$ with the same strength for all four tasks. From 0s to $5 \mathrm{~s}$, there was sustained stimulation on both wrists, while the subjects performed different SS tasks. This resulted in distinctive oscillatory changes across the left and right somatosensory cortex. During the SS-L task, the ERD on the contralateral (right side) hemisphere $\mathrm{C} 4$ was more pronounced than that in the ipsilateral (left side) hemisphere C3. Conversely, during the SS-R task, the ERD in C3 was more pronounced than that in $\mathrm{C} 4$; during SS-B, a clear bilateral activation was presented; during the SS-S task, the ERD strength was much lower bilaterally than for all other tasks. Moreover, Fig. 3 shows the grand-averaged ERD/ERS spatial distribution during different tasks in alpha frequency band. It can been seen that in Fig. 3 (5) the vibration burst resulted in
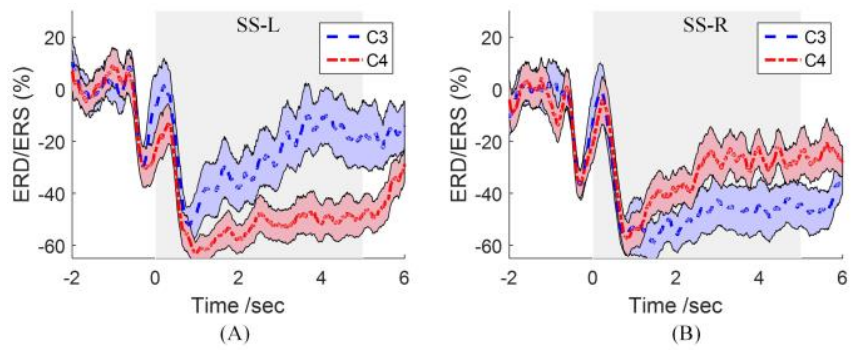

(B)

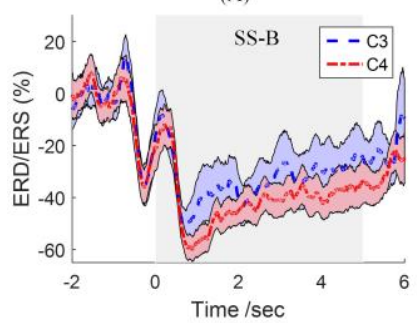

(C)

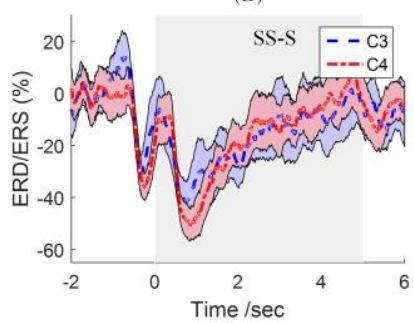

(D)
Figure 2. The time varying grand-averaged ERD/ERS curves at small-Laplace filtered $\mathrm{C} 3$ and $\mathrm{C} 4$ channels within alpha-beta frequency band [8 26] Hz. (A) ERD/ERS corresponds to SS-L task. (B) ERD/ERS corresponds to SS-R task. (C) ERD/ERS corresponds to SS-B task. (D) ERD/ERS corresponds to SS-S task. The upper and lower curves indicate standard error. Time 0s corresponds to the time when the indicating cue appeared (3rd second from the beginning of the trial). 


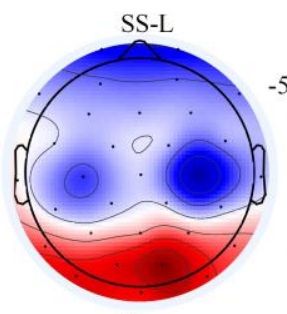

(1)

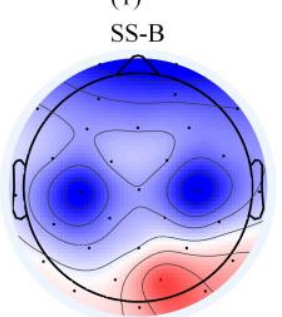

(3)

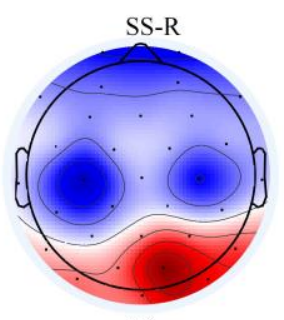

(2)

SS-S

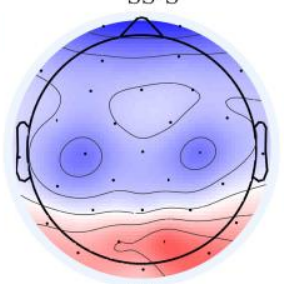

(4)
Figure 3. Grand-averaged ERD/ERS distribution within alpha frequency band [8 13] Hz. (1) ERD/ERS activation with respect to SS-L task. (2) ERD/ERS activation with respect to SS-R task. (3) ERD/ERS activation with respect to SS-B task. (4) ERD/ERS activation with respect to SS-S task. (5) ERD/ERS activation with respect to vibration burst (1 second before the appearance of the cue). Color bar indicates the ERD/ERS value. Note: ERD/ERS value is averaged between 1 to 4 second after the appearance of the cue in subfigure (1) (2) (3) (4); ERD/ERS value is averaged between -0.5 to 0 second before the appearance of the cue in (5).

left and right somatosensory cortex co-activation, and it was clearly concentrated on left (C3) and right (C4) hemispheres. A clear occipital ERS was also present during this vibration burst ready period. There was a clear somatosensory activation during the sustained tactile stimulation (Fig. 3 (1) (2) (3) (4)); while different SS tasks resulted in distinctive cortical activation distributions, with contralateral stronger activation during both SS-L and SS-R task, with bilateral activation

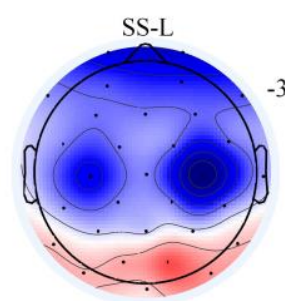

(1)

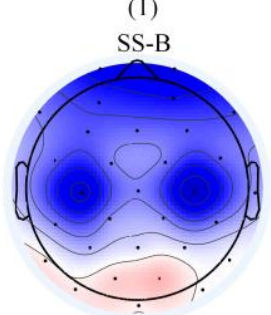

(3)

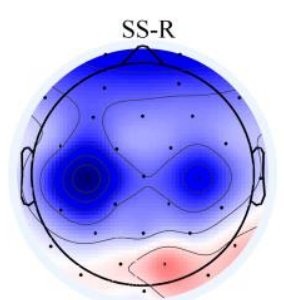

(2)

SS-S

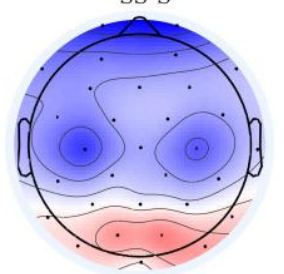

(4)
Figure 4. Grand-averaged ERD/ERS distribution within alpha-beta frequency band [8 26] Hz. (1) ERD/ERS activation with respect to SS-L task. (2) ERD/ERS activation with respect to SS-R task. (3) ERD/ERS activation with respect to SS-B task. (4) ERD/ERS activation with respect to SS-S task. (5) ERD/ERS activation with respect to vibration burst (1 second before the appearance of the cue). Color bar indicates the ERD/ERS value. Note: ERD/ERS value is averaged between 1 to 4 second after the appearance of the cue in subfigure (1) (2) (3) (4); ERD/ERS value is averaged between -0.5 to 0 second before the appearance of the cue in (5).

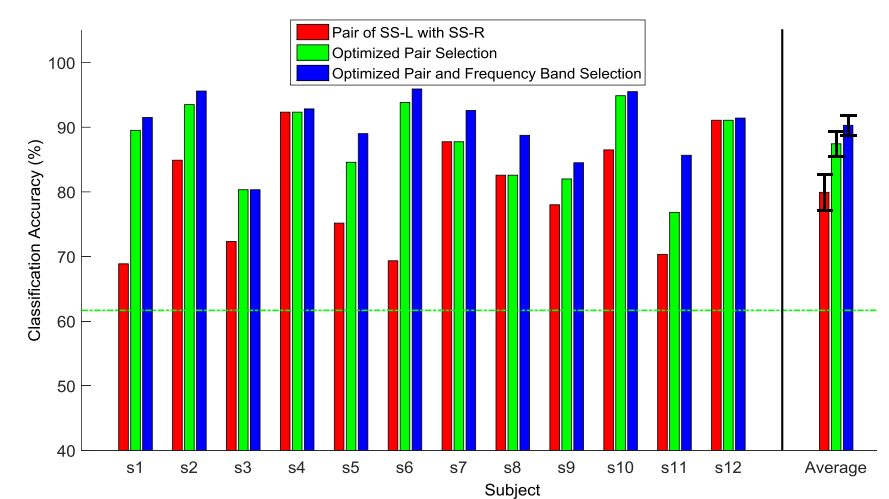

Figure 5. BCI performance in two class tactile BCI systems. The red bars indicate the BCI performance based SS-L and SS-R, which we proposed in [34], [36]; the green bars indicate the BCI performance with the optimal task pair for each subject; and the blue bars indicate the BCI performance with both task pair and frequency band optimization for each subject. The green dash-dotted line indicates $61.67 \%$ corrected chance level with $\mathrm{p}=\mathbf{0 . 0 5}$ according to $[48]$.

during SS-B task and suppressed activation during SS-S task. Moreover, occipital suppression (ERS) was shown in all SS tasks, with stronger ERS in both SS-L and SS-R tasks than that in SS-B and SS-S tasks. Moreover, grand-averaged ERD/ERS spatial distribution in alpha-beta frequency band $\left(\left[\begin{array}{l}8 \\ 26\end{array}\right] \mathrm{Hz}\right)$ is shown in Fig. 4.

\section{B. Two-Class Scenarios}

Table 1 summarized the two-class performance of the proposed tactile BCI system. With a common frequency band of [ 826$] \mathrm{Hz}$, the average accuracy was $79.9 \pm 8.7 \%$ for P1 (SS-R vs SS-L), $73.8 \pm 8.0 \%$ for $\mathrm{P} 2,83.4 \pm 5.6 \%$ for $\mathrm{P} 3,69.6 \pm 5.6 \%$ for $\mathrm{P} 4,81.3 \pm 10.0 \%$ for $\mathrm{P} 5,75.1 \pm 10.8 \%$ for P6. Without any subject-specific optimization, one-way ANOVA with repeated measures revealed a significant difference in classification accuracy among different pairs $(p<0.05)$. Post-hoc testing showed that P1, P3 and P5 were significantly better that P4, and no significant difference was found among P1, P3 and P5. If the best two-class task pair for individual subjects was used (optimized pair selection in Fig. 5), the average classification accuracy increased to $87.4 \pm 6.0 \%$ ( $\mathrm{p}<0.05)$, with the highest subject above $95 \%$. If the frequency bands were optimized for individual subjects, the average accuracy further increased to $90.3 \pm 4.9 \%$. Fig. 6 illustrates an example of differences in discriminative information $\mathrm{R}^{2}$ distribution of the best and the

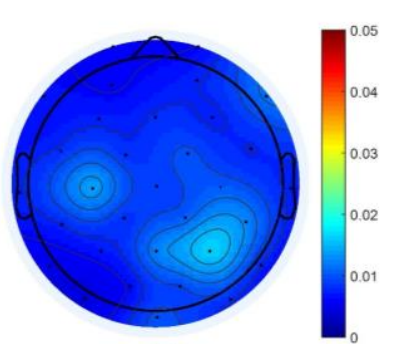

(A)

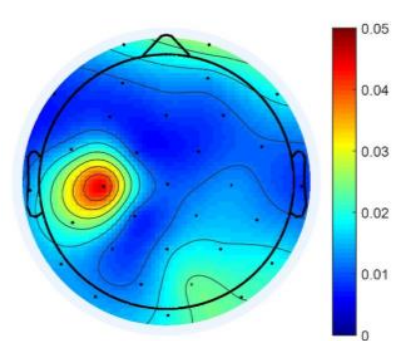

(B)
Figure 6. $R^{2}$ value distribution of subject $s 1$. (A) $R^{2}$ value distribution of task pair P1 (SS-L vs SS-R); (B) $R^{2}$ value distribution of task pair P5 (SS-R vs SS-S). The $R^{2}$ value was averaged between 1 to 4 second from the appearance of the cue, within 10 and $13 \mathrm{~Hz}$ frequency band. The clear advantage in discriminative power of $P 5$ over $P 1$ is readily seen. 
Table 1. Classification accuracy between each pair of the SS tasks. The first column of each pair (D1) indicates the accuracy in [8 26] Hz frequency band, whereas the second column (D2) indicates optimized accuracy, and brackets indicate subject optimized frequency band. And the entries in bold-face indicate the case with the highest accuracy for each subject when both the task pairs and frequency bands are optimized.

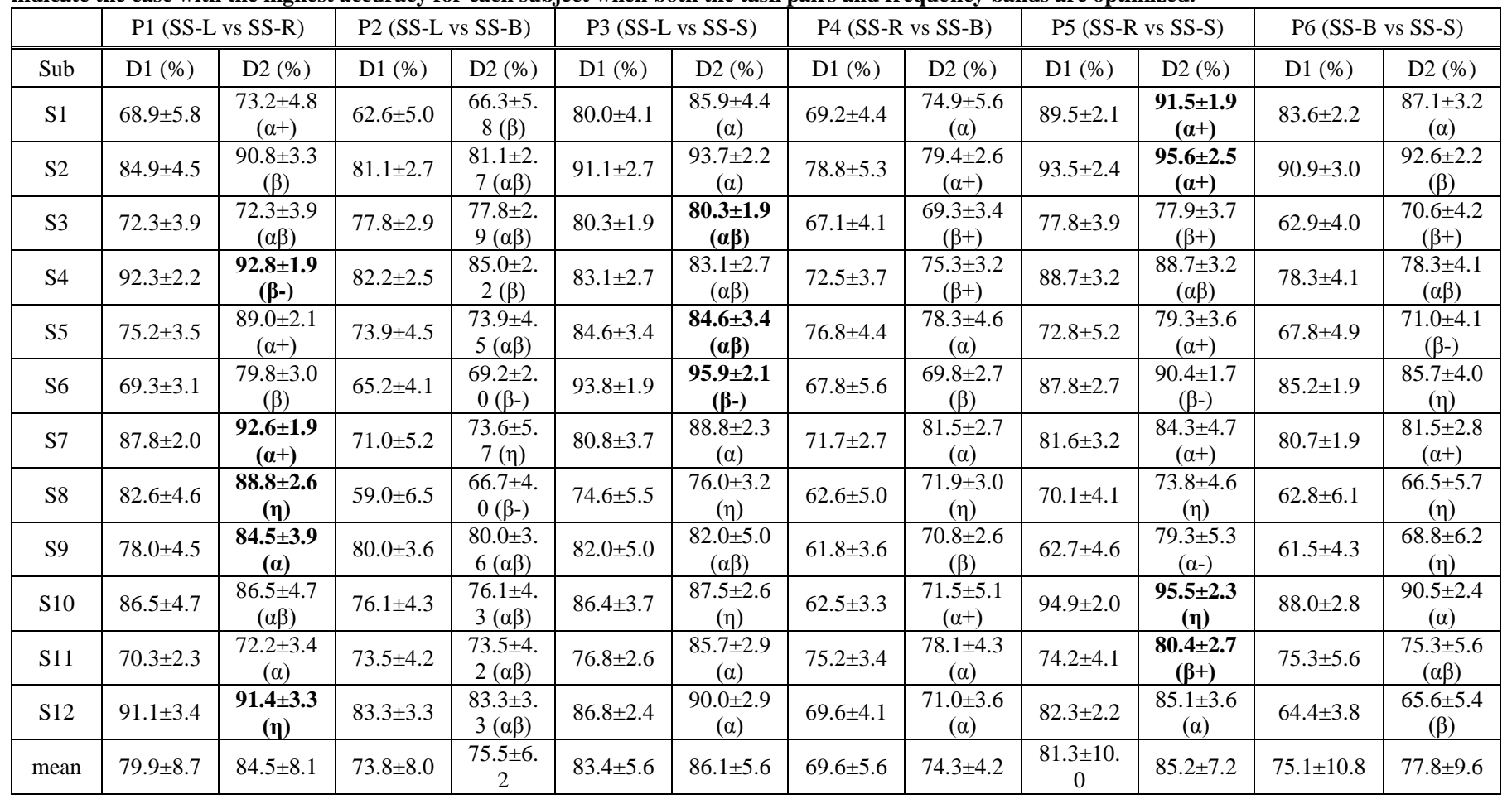

worst task pair of a subject. A sharp contrast can be observed. This indicates that a subject-specific selection of the best SS task pair can significantly enhance the performance of the SS tactile BCI. Interestingly, even for the basic SS-L to SS-R scenario, 10 out of the 12 subjects exceeded $70 \%$ accuracy and all of them were above the corrected channel level of $61.67 \%$ with $\mathrm{p}=0.05$. With subject optimal frequency band selection and/or task pair selection, accuracy was above $70 \%$ in all cases (Fig. 5).

\section{Three- and Four-class Scenarios}

Table 2 summarized the three-class performance of the proposed tactile BCI system. With a common frequency band of [ $\left.\begin{array}{ll}8 & 26\end{array}\right] \mathrm{Hz}$, the average accuracy was $61.8 \pm 7.4 \%$ for $\mathrm{T} 1$,

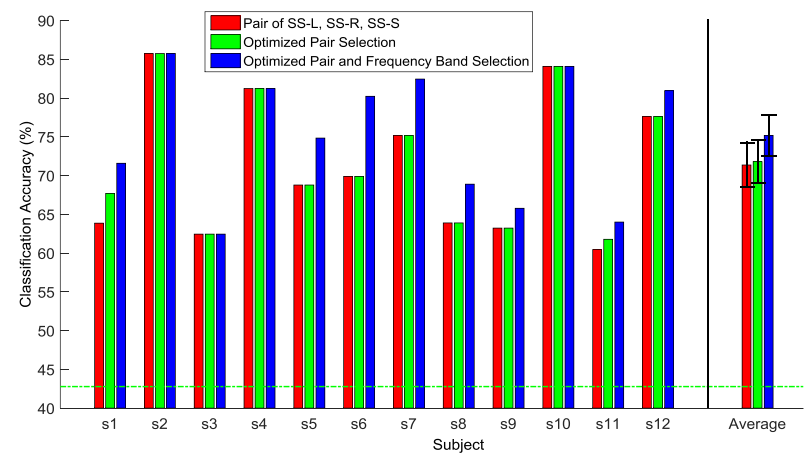

Figure 7. BCI performance in three-class tactile BCI systems. The red bars indicate the BCI performance based left hand SS, right hand SS, and suppressed SS tasks; the green bars indicate the BCI performance with the optimal task set for each subject; and the blue bars indicate the BCI performance with both task set and frequency band optimization for each subject. The green dash-dotted line indicates $42.78 \%$ corrected chance level with $p=0.05$.
$71.4 \pm 9.1 \%$ for $\mathrm{T} 2,64.6 \pm 7.6 \%$ for $\mathrm{T} 3,62.2 \pm 8.8 \%$ for $\mathrm{T} 4$. For these three-class scenarios, one-way ANOVA with repeated measures showed that there was a significant difference in classification accuracy $(p<0.05)$, and post-hoc comparison showed that the performance of $\mathrm{T} 2$ was significantly greater than the other three scenarios, and no significant difference was found among T1, T3 and T4. Fig. 7 illustrates the performance of the three-class tactile BCI system. All subjects exceeded the corrected random chance level of $42.78 \% \quad(p=0.05)$, and reached an averaged accuracy of $71.4 \pm 9.1 \%$. The selection of the optimal pair did not show substantial improvements from the original T2. However, subject specific frequency band selection improved the three-class accuracy to $75.2 \pm 8.3 \%$ (paired-t test; $p<0.05$ ).

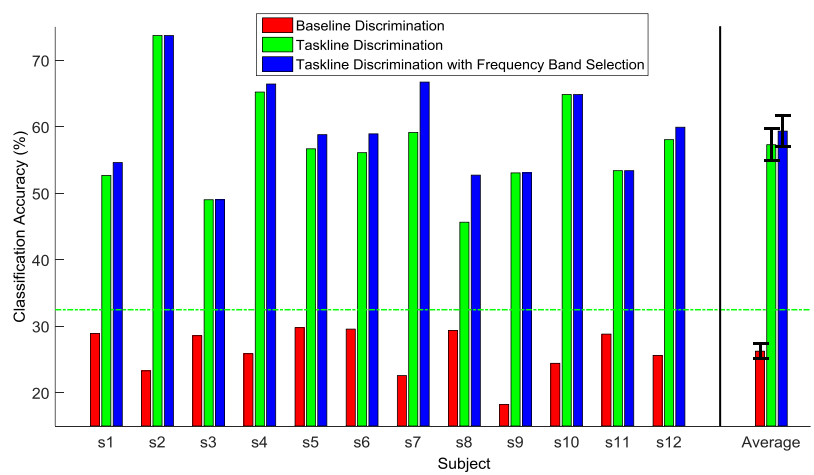

Figure 8. BCI performance in four class tactile BCI systems. The red bars indicate the BCI performance within the baseline time period; the green bars indicate the BCI performance in taskline period; and the blue bars indicate the BCI performance with optimization of frequency band for each subject. The green dash-dotted line indicates $32.50 \%$ corrected chance level with $\mathbf{p}=\mathbf{0 . 0 5}$. 
Table 2. Classification accuracy among every three SS tasks. The first column of each pair (D1) indicates the accuracy in [8 26] Hz frequency band, whereas the second column (D2) indicates optimized accuracy, and brackets indicate subject optimized frequency band. And the entries in bold-face indicate the case with the highest accuracy for each subject when both the task pairs and frequency bands are optimized.

\begin{tabular}{|c|c|c|c|c|c|c|c|c|}
\hline & T1 (SS-L vs SS-R vs SS-B) & T2 (SS-L vs SS-R vs SS-S) & \multicolumn{2}{|c|}{ T3 (SS-L vs SS-B vs SS-S) } & \multicolumn{2}{c|}{ T4 (SS-R vs SS-B vs SS-S) } \\
\hline Subj & D1 $(\%)$ & D2 $(\%)$ & D1 $(\%)$ & D2 $(\%)$ & D1 $(\%)$ & D2 $(\%)$ & D1 $(\%)$ & D2 $(\%)$ \\
\hline S1 & $53.8 \pm 2.6$ & $53.8 \pm 2.6(\alpha \beta)$ & $63.9 \pm 4.5$ & $69.0 \pm 4.0(\alpha+)$ & $62.5 \pm 3.8$ & $62.5 \pm 3.8(\alpha \beta)$ & $67.7 \pm 4.1$ & $\mathbf{7 1 . 6} \pm \mathbf{5 . 3}(\boldsymbol{\alpha})$ \\
\hline S2 & $73.2 \pm 3.2$ & $73.2 \pm 3.2(\alpha \beta)$ & $85.7 \pm 3.3$ & $\mathbf{8 5 . 7} \pm \mathbf{3 . 3}(\boldsymbol{\alpha} \boldsymbol{\beta})$ & $79.3 \pm 4.2$ & $79.3 \pm 4.2(\alpha \beta)$ & $79.0 \pm 3.2$ & $79.6 \pm 2.9(\alpha)$ \\
\hline S3 & $60.7 \pm 4.7$ & $60.7 \pm 4.7(\alpha \beta)$ & $62.4 \pm 1.9$ & $\mathbf{6 2 . 4} \pm \mathbf{1 . 9}(\boldsymbol{\alpha} \boldsymbol{\beta})$ & $57.3 \pm 4.5$ & $59.3 \pm 4.1(\beta+)$ & $53.1 \pm 2.7$ & $59.1 \pm 1.6(\beta+)$ \\
\hline S4 & $70.7 \pm 2.1$ & $73.1 \pm 3.2(\beta)$ & $81.2 \pm 3.7$ & $\mathbf{8 1 . 2} \pm \mathbf{3 . 7}(\boldsymbol{\alpha} \boldsymbol{\beta})$ & $68.8 \pm 2.8$ & $70.3 \pm 3.1(\beta)$ & $68.3 \pm 1.9$ & $68.6 \pm 3.4(\beta+)$ \\
\hline S5 & $66.6 \pm 2.8$ & $66.6 \pm 2.8(\alpha \beta)$ & $68.8 \pm 3.0$ & $\mathbf{7 4 . 8} \pm \mathbf{2 . 8}(\boldsymbol{\alpha}+)$ & $63.5 \pm 2.3$ & $63.5 \pm 2.3(\alpha \beta)$ & $59.3 \pm 2.5$ & $62.9 \pm 4.4(\alpha)$ \\
\hline S6 & $49.2 \pm 3.4$ & $59.4 \pm 3.6(\beta)$ & $69.9 \pm 2.8$ & $\mathbf{8 0 . 2} \pm \mathbf{3 . 2}(\boldsymbol{\beta})$ & $67.5 \pm 3.0$ & $68.9 \pm 2.3(\beta-)$ & $68.4 \pm 4.1$ & $68.4 \pm 4.1(\alpha \beta)$ \\
\hline S7 & $63.3 \pm 3.5$ & $70.2 \pm 3.8(\alpha)$ & $75.2 \pm 2.8$ & $\mathbf{8 2 . 4} \pm \mathbf{2 . 4}(\boldsymbol{\alpha}+)$ & $64.3 \pm 2.3$ & $71.2 \pm 2.9(\alpha+)$ & $67.2 \pm 3.9$ & $72.4 \pm 3.4(\alpha)$ \\
\hline S8 & $52.1 \pm 3.2$ & $58.3 \pm 3.2(\eta)$ & $63.9 \pm 3.1$ & $\mathbf{6 8 . 9} \mathbf{3} .7(\boldsymbol{\eta})$ & $51.4 \pm 4.5$ & $55.6 \pm 3.3(\eta)$ & $50.1 \pm 2.4$ & $58.2 \pm 3.9(\eta)$ \\
\hline S9 & $62.3 \pm 2.7$ & $62.9 \pm 5.9(\beta)$ & $63.2 \pm 5.2$ & $\mathbf{6 5 . 8} \pm \mathbf{6 . 6}(\boldsymbol{\alpha})$ & $58.9 \pm 2.3$ & $61.3 \pm 4.2(\eta)$ & $50.0 \pm 4.1$ & $52.7 \pm 4.6(\beta-)$ \\
\hline S10 & $60.7 \pm 4.1$ & $60.7 \pm 4.3(\alpha+)$ & $84.1 \pm 3.0$ & $\mathbf{8 4 . 1} \pm \mathbf{3 . 0}(\boldsymbol{\alpha} \boldsymbol{\beta})$ & $75.4 \pm 4.1$ & $76.3 \pm 3.6(\eta)$ & $66.6 \pm 4.3$ & $74.0 \pm 3.0(\alpha+)$ \\
\hline S11 & $60.4 \pm 3.6$ & $61.2 \pm 3.1(\alpha)$ & $60.4 \pm 4.7$ & $\mathbf{6 4 . 0} \pm \mathbf{3 . 1}(\boldsymbol{\beta})$ & $61.8 \pm 3.3$ & $61.8 \pm 3.3(\alpha \beta)$ & $59.5 \pm 2.1$ & $59.9 \pm 3.5(\beta+)$ \\
\hline S12 & $69.1 \pm 5.1$ & $69.1 \pm 5.1(\alpha \beta)$ & $77.6 \pm 4.6$ & $\mathbf{8 0 . 9} \pm \mathbf{2 . 5}(\boldsymbol{\alpha})$ & $63.9 \pm 3.2$ & $64.7 \pm 2.5(\eta)$ & $57.1 \pm 3.7$ & $58.5 \pm 2.5(\alpha)$ \\
\hline mean & $61.8 \pm 7.4$ & $64.1 \pm 6.2$ & $71.4 \pm 9.1$ & $75.0 \pm 8.5$ & $64.6 \pm 7.6$ & $66.2 \pm 7.0$ & $62.2 \pm 8.8$ & $65.5 \pm 8.1$ \\
\hline
\end{tabular}

Fig. 8 illustrates the performance of the proposed four-class SS tactile BCI system. The task activity discrimination accuracy was compared against the baseline performance, when no tasks were performed. The proposed system achieved an average accuracy of $59.4 \pm 7.3 \%$, and all subjects exceeded the corrected random chance level of $32.50 \%(p=0.05)$. For this scenario, subject-specific frequency band selection resulted in a small (but significant) improvement of $2 \%(p<0.05)$.

\section{DISCUSSION}

We presented a novel approach for a multiple-class tactile BCI based on stimulus-induced oscillatory dynamics. This is the first example of a multi-class BCI based on somatosensory oscillations. The proposed SS tactile BCI approach is different from existing tactile BCI systems based on SSSEP [25] or event-related potentials [33]. The present system does not need the user to engage his/her visual attention, which is vital for patients without eye control. Moreover, the intrinsic low SNR of SSSEP led to a relatively low BCI performance and relatively high BCI-illiteracy rate in previous systems. An alternative tactile BCI system is the tactile P300 system, which is based on the oddball paradigm, and was shown to allow an accuracy of approximately $72 \%$. In the current study, we took a different approach in exploring EEG dynamics associated with tactile stimulation. We showed that the stimulus-induced oscillatory dynamics provides a powerful approach for high-performance tactile BCI systems. In the two-class scenarios, the mean classification accuracy of P1 was approximately $80 \%$, in agreement with the results from our previous studies [34], [36]. With subject-specific task pair and frequency band optimization, however, the performance of the two-class classification reached approximately 90\%. This accuracy level is substantially greater than any tactile BCI reported in the literature, and is among the best among current two-class BCIs [25], [33], [49], [50]. Even in the worst case scenario (without task pair and frequency band selection), 10 out of 12 subjects exceeded the $70 \%$ threshold in accuracy. This indicates potential for the proposed system to be used by a large portion of the population.

Combining the SSSEP and transient ERP signal modalities, a hybrid three-class tactile BCI was recently proposed by Breitwieser et al. [26], with an average accuracy of 57\%. In our current experimental evaluation, we showed an accuracy of $75.2 \%$ for a three-class scenario. Thus, in multiple-class BCI settings, the stimulus-induced oscillatory dynamics provides extra information for somatosensory attention decoding and has a higher SNR as compared to SSSEP and tERP. Moreover, our four-class tactile BCI system showed an average accuracy of $59.4 \%$, thus making it a promising approach for multiple-class BCI development. The SSSEP response reflects the somatic information processing. It has a frequency specific feature, which is related to the stimulation frequency [27], [31]. In contrast, the ERD/ERS oscillatory dynamics reflects somatosensory processing, and has a non-stimulation frequency specific feature [51]. Therefore, the ERD/ERS oscillatory response and SSSEP response provide complementary information of the somatosensory input processing. Thus theoretically, in tactile BCIs, hybridizing the oscillatory dynamics and SSSEP response will provide a way to improve the BCI performance.

It was clearly observed that the vibration burst for alerting subjects to get ready for the subsequent task induced an oscillatory ERD response in the [8 26] $\mathrm{Hz}$ alpha and beta frequency bands in both the left and right hemisphere (Fig. 2 and Fig. 3 (5)), but it did not exhibit task-related differences as no specific tasks were performed. During sustained tactile stimulation in task periods, ERD/ERS oscillatory activation showed task-related differences in the left and right hemisphere. In SS-S tasks, the subjects were instructed to actively ignore or suppress the tactile sensation (idle state), thus the activation on both hemispheres represented mainly the stimulus effect. In contrast during SS-B tasks, the subjects were instructed to focus the sensation on both hands. In this case, the ERD oscillatory activation was much more pronounced than 
that during the SS-S task, and the activation difference between SS-S and SS-B represented the active somatosensory attention involvement. In SS-L and SS-R tasks, it has been shown that the contralateral activation was stronger than that in ipsilateral activation, which was in accordance with our previously published results [36] and further supported our proposal of a novel tactile BCI based on stimulus-induced oscillatory dynamics. The frequency band of [8 26] Hz covered most of the stimulus-induced oscillatory components (alpha, beta), and was independent from the stimulation frequency specific SSSEP [25]. The stimulation pattern was the same during all the proposed four tactile sensation tasks. Therefore, the selection of [8 26] Hz would not influence current results. Moreover, the BCI performance of [ [8 30] Hz including both 23 and $27 \mathrm{~Hz}$ frequency did not shown significant difference from current $[8$ 26] $\mathrm{Hz}$ frequency band, which included $23 \mathrm{~Hz}$ frequency only.

ERD/ERS oscillatory changes are not only correlated with real movement or imagined movement [52], [53], but also with tactile sensory processing [38]. In our previous study [18], these oscillatory dynamics were systematically compared between the motor imagery and sensory stimulation, showing that MI and SS shared a similar activation pattern and ERD/ERS dynamics although the brain activation sources were different (one is generated mainly from the motor cortex, while the other from the somatosensory cortex). In this experimental study, the subjects were instructed to selectively focus their somatosensory attention on one hand at a time while both wrists were simultaneously stimulated. SS-L and SS-R both showed stronger contralateral activation, which were similar to motor imagery, but from a different brain region (somatosensory cortex). Oscillatory signals from the somatosensory cortex would provide alternative opportunities for $\mathrm{BCI}$ design, when the motor cortex is impaired. The somatosensory-BCI [36] would also provide a promising signal for neurorehabilitation following stroke.

In this experimental design, the subjects were instructed to perform the four selective sensation tasks according to their respective cues, which were randomly presented. In order to limit the subject's potential mental adjustment while performing the tasks in an online scheme, no on-line feedback was presented to the subjects in this proof-of-concept study. However, the results from offline analysis for the two-class classification were comparable with our previous results obtained during an online protocol. In this study, the stimulation parameters were slightly different across subjects due to different wrist size. Such difference have shown to have limited influence for current tactile BCI performance and ERD/ERS dynamics [34], [35], [54]. Stimuli parameters, such as amplitude and frequency, and their correlation with ERD/ERS dynamics could be the topic of a future study, with the goal of further enhancing the performance tactile BCI.

\section{CONCLUSION}

A tactile BCI based on stimulus-induced oscillatory dynamics (selective sensation based tactile BCI), results in a significant improvement in detection accuracy compared to current tactile BCI designs, that are based on either steady-state somatosensory evoked or event related potentials. The oscillatory activation across the four tactile sensation tasks (SS-L, SS-R, SS-B, SS-S) exhibited distinct dynamic processes between the somatosensory attention and stimulus-induced oscillation, which provides a novel approach for a high-performance multiple-class tactile BCI setup. This tactile $\mathrm{BCI}$ design provides a novel approach for enhancing the current tactile BCIs based on SSSEP and ERP, reducing BCI-illiteracy users, and offering more commands.

\section{ACKNOWLEDGEMENT}

We thank all volunteers for their participation in the study. This work is supported by the University Starter Grant of the University of Waterloo (No. 203859), the National Natural Science Foundation of China (Grant No. 51620105002, 51375296, 51421092), the Research Project of State Key Laboratory of Mechanical System and Vibration MSV201607.

\section{REFERENCE}

[1] J. R. Wolpaw, N. Birbaumer, D. J. McFarland, G. Pfurtscheller, T. M. Vaughan, and others, "Brain-computer interfaces for communication and control," Clin. Neurophysiol., vol. 113, no. 6, pp. 767-791, 2002.

[2] J. J. Daly and J. R. Wolpaw, "Brain--computer interfaces in neurological rehabilitation," Lancet Neurol., vol. 7, no. 11, pp. 1032-1043, 2008.

[3] J. R. Wolpaw and D. J. McFarland, "Control of a two-dimensional movement signal by a noninvasive brain-computer interface in humans," Proc. Natl. Acad. Sci. U. S. A., vol. 101, no. 51, pp. 17849-17854, 2004.

[4] G. Pfurtscheller and C. Neuper, "Motor imagery and direct brain-computer communication," Proc. IEEE, vol. 89, no. 7, pp. 1123-1134, 2001.

[5] G. Pfurtscheller, "Spatiotemporal ERD/ERS patterns during voluntary movement and motor imagery," Suppl. Clin. Neurophysiol., vol. 53, pp. 196-198, 2000.

[6] G. Pfurtscheller, C. Neuper, C. Brunner, F. L. da Silva, and others, "Beta rebound after different types of motor imagery in man.," Neurosci. Lett., vol. 378, no. 3, p. 156, 2005.

[7] B. Blankertz, K.-R. Muller, G. Curio, T. M. Vaughan, G. Schalk, J. R. Wolpaw, A. Schlogl, C. Neuper, G. Pfurtscheller, T. Hinterberger, and others, "The BCI competition 2003: progress and perspectives in detection and discrimination of EEG single trials," Biomed. Eng. IEEE Trans., vol. 51, no. 6, pp. 1044-1051, 2004.

[8] B. Blankertz, K.-R. Muller, D. J. Krusienski, G. Schalk, J. R. Wolpaw, A. Schlogl, G. Pfurtscheller, J. R. Millan, M. Schroder, and N. Birbaumer, "The BCI competition III: Validating alternative approaches to actual BCI problems," Neural Syst. Rehabil. Eng. IEEE Trans., vol. 14, no. 2, pp. 153-159, 2006.

[9] M. Tangermann, K.-R. Müller, A. Aertsen, N. Birbaumer, C. Braun, C. Brunner, R. Leeb, C. Mehring, K. J. Miller, G. R. Müller-Putz, and others, "Review of the BCI competition IV," Front. Neurosci., vol. 6, 2012.

[10] A. J. Doud, J. P. Lucas, M. T. Pisansky, and B. He, "Continuous three-dimensional control of a virtual helicopter using a motor imagery based brain-computer interface," PLoS One, vol. 6, no. 10, p. e26322, 2011.

[11] V. Kaiser, G. Bauernfeind, A. Kreilinger, T. Kaufmann, A. Kübler, C. Neuper, and G. R. Müller-Putz, "Cortical effects of user training in a motor imagery based brain-computer interface measured by fNIRS and EEG.," Neuroimage, vol. 85 Pt 1, pp. 432-44, Jan. 2014.

[12] F. Pichiorri, F. De Vico Fallani, F. Cincotti, F. Babiloni, M. Molinari, S. C. Kleih, C. Neuper, a Kübler, and D. Mattia, "Sensorimotor rhythm-based brain-computer interface training: the impact on motor cortical responsiveness.," J. Neural Eng., vol. 8, no. 2, p. 25020, Apr. 2011.

[13] C. Guger, G. Edlinger, W. Harkam, I. Niedermayer, and G. Pfurtscheller, "How many people are able to operate an EEG-based 
brain-computer interface (BCI)?," Neural Syst. Rehabil. Eng. IEEE Trans., vol. 11, no. 2, pp. 145-147, 2003.

[14] C. Guger, S. Daban, E. Sellers, C. Holzner, G. Krausz, R. Carabalona, F. Gramatica, and G. Edlinger, "How many people are able to control a P300-based brain--computer interface (BCI)?," Neurosci. Lett., vol. 462, no. 1, pp. 94-98, 2009.

[15] C. Vidaurre, C. Sannelli, K.-R. Müller, and B. Blankertz, "Co-adaptive calibration to improve BCI efficiency," J. Neural Eng., vol. 8, no. 2, p. 25009, 2011.

[16] C. Vidaurre and B. Blankertz, "Towards a cure for BCI illiteracy," Brain Topogr., vol. 23, no. 2, pp. 194-198, 2010.

[17] B. Z. Allison, C. Brunner, V. Kaiser, G. R. Müller-Putz, C. Neuper, and G. Pfurtscheller, "Toward a hybrid brain--computer interface based on imagined movement and visual attention," J. Neural Eng., vol. 7, no. 2, p. 26007, 2010.

[18] L. Yao, J. Meng, X. Sheng, D. Zhang, and X. Zhu, "A novel calibration and task guidance framework for motor imagery BCI via a tendon vibration induced sensation with kinesthesia illusion.," $J$. Neural Eng., vol. 12, no. 1, p. 16005, Dec. 2015.

[19] F. Nijboer, E. W. Sellers, J. Mellinger, M. a Jordan, T. Matuz, a Furdea, S. Halder, U. Mochty, D. J. Krusienski, T. M. Vaughan, J. R. Wolpaw, N. Birbaumer, and a Kübler, "A P300-based brain-computer interface for people with amyotrophic lateral sclerosis.," Clin. Neurophysiol., vol. 119, no. 8, pp. 1909-16, Aug. 2008.

[20] M. Cheng, X. Gao, S. Gao, and D. Xu, "Design and implementation of a brain-computer interface with high transfer rates," Biomed. Eng. IEEE Trans., vol. 49, no. 10, pp. 1181-1186, 2002.

[21] A. R. Murguialday, J. Hill, M. Bensch, S. Martens, S. Halder, F. Nijboer, B. Schoelkopf, N. Birbaumer, and A. Gharabaghi, "Transition from the locked in to the completely locked-in state: a physiological analysis.," Clin. Neurophysiol., vol. 122, no. 5, pp. 925-33, May 2011.

[22] a Kübler and N. Birbaumer, "Brain-computer interfaces and communication in paralysis: extinction of goal directed thinking in completely paralysed patients?," Clin. Neurophysiol., vol. 119, no. 11, pp. 2658-66, Nov. 2008.

[23] D. De Massari, C. a Ruf, A. Furdea, T. Matuz, L. van der Heiden, S. Halder, S. Silvoni, and N. Birbaumer, "Brain communication in the locked-in state.," Brain, vol. 136, no. Pt 6, pp. 1989-2000, Jun. 2013. N. Birbaumer and L. G. Cohen, "Brain--computer interfaces: communication and restoration of movement in paralysis," $J$. Physiol., vol. 579, no. 3, pp. 621-636, 2007.

[25] G. R. Müller-Putz, R. Scherer, C. Neuper, and G. Pfurtscheller, "Steady-state somatosensory evoked potentials: Suitable brain signals for brain-computer interfaces?," IEEE Trans. Neural Syst. Rehabil. Eng., vol. 14, no. 1, pp. 30-37, 2006.

[26] C. Breitwieser, C. Pokorny, and G. R. Müller-Putz, "A hybrid three-class brain-computer interface system utilizing SSSEPs and transient ERPs," J. Neural Eng., vol. 13, no. 6, p. 66015, 2016. S. Tobimatsu, Y. M. Zhang, and M. Kato, "Steady-state vibration somatosensory evoked potentials: physiological characteristics and tuning function," Clin. Neurophysiol., vol. 110, no. 11, pp. 1953-1958, Nov. 1999.

[28] M. Severens, J. Farquhar, P. Desain, J. Duysens, and C. Gielen, "Transient and steady-state responses to mechanical stimulation of different fingers reveal interactions based on lateral inhibition," Clin. Neurophysiol., vol. 121, no. 12, pp. 2090-2096, 2010.

[29] C. Nangini, B. Ross, F. Tam, and S. J. Graham, "Magnetoencephalographic study of vibrotactile evoked transient and steady-state responses in human somatosensory cortex," Neuroimage, vol. 33, no. 1, pp. 252-262, 2006.

[30] C. Breitwieser, V. Kaiser, C. Neuper, and G. R. Müller-Putz, "Stability and distribution of steady-state somatosensory evoked potentials elicited by vibro-tactile stimulation.," Med. Biol. Eng. Comput., vol. 50, no. 4, pp. 347-57, Apr. 2012.

[31] C.-M. Giabbiconi, N. J. Trujillo-Barreto, T. Gruber, and M. M. Müller, "Sustained spatial attention to vibration is mediated in primary somatosensory cortex.," Neuroimage, vol. 35, no. 1, pp. 255-62, Mar. 2007.

[32] S. Ahn, M. Ahn, H. Cho, and S. Chan Jun, "Achieving a hybrid brain-computer interface with tactile selective attention and motor imagery," J. Neural Eng., vol. 11, no. 6, p. 66004, Dec. 2014.

[33] A.-M. Brouwer and J. B. F. Van Erp, "A tactile P300 brain-computer interface," Front. Neurosci., vol. 4, p. 19, 2010.
[34] L. Yao, J. Meng, D. Zhang, X. Sheng, and X. Zhu, "Selective Sensation Based Brain-Computer Interface via Mechanical

Vibrotactile Stimulation," PLoS One, vol. 8, no. 6, 2013.

[35] L. Yao, J. Meng, D. Zhang, X. Sheng, and X. Zhu, "Combining motor imagery with selective sensation toward a hybrid-modality BCI," IEEE Trans. Biomed. Eng., vol. 61, no. 8, pp. 2304-2312, 2014.

[36] L. Yao, X. Sheng, D. Zhang, N. Jiang, D. Farina, and X. Zhu, "A BCI System based on Somatosensory Attentional Orientation," IEEE Trans. Neural Syst. Rehabil. Eng., vol. 4320, no. c, pp. 1-1, 2016.

[37] N. Sharma, V. M. Pomeroy, and J.-C. Baron, "Motor imagery: a backdoor to the motor system after stroke?," Stroke., vol. 37, no. 7, pp. 1941-52, Jul. 2006

[38] E. Houdayer, a Degardin, J. Salleron, J. L. Bourriez, L. Defebvre, F. Cassim, and P. Derambure, "Movement preparation and cortical processing of afferent inputs in cortical tremor: an event-related (de)synchronization (ERD/ERS) study.," Clin. Neurophysiol., vol. 123, no. 6, pp. 1207-15, Jun. 2012.

[39] C. Dockstader, D. Cheyne, and R. Tannock, "Cortical dynamics of selective attention to somatosensory events.," Neuroimage, 2010.

[40] C. Breitwieser, V. Kaiser, C. Neuper, and G. R. Müller-Putz, "Stability and distribution of steady-state somatosensory evoked potentials elicited by vibro-tactile stimulation," Med. Biol. Eng. Comput., pp. 1-11, 2012.

[41] B. Graimann, J. E. Huggins, S. P. Levine, and G. Pfurtscheller, "Visualization of significant ERD/ERS patterns in multichannel EEG and ECoG data," Clin. Neurophysiol., vol. 113, no. 1, pp. 43-47, 2002.

[42] A. Delorme, T. Mullen, C. Kothe, Z. A. Acar, N. Bigdely-Shamlo, A. Vankov, and S. Makeig, "EEGLAB, SIFT, NFT, BCILAB, and ERICA: new tools for advanced EEG processing," Comput. Intell. Neurosci., vol. 2011, p. 10, 2011.

[43] R. Oostenveld, P. Fries, E. Maris, and J. M. Schoffelen, "FieldTrip: open source software for advanced analysis of MEG, EEG, and invasive electrophysiological data," Comput. Intell. Neurosci., vol. 2011, p. 1, 2011.

[44] T. H. Wonnacott and R. J. Wonnacott, Introductory statistics, vol. 19690. Wiley Chichester, 1990.

[45] G. Schalk, D. J. McFarland, T. Hinterberger, N. Birbaumer, and J. R. Wolpaw, "BCI2000: a general-purpose brain-computer interface (BCI) system," Biomed. Eng. IEEE Trans., vol. 51, no. 6, pp. 1034-1043, 2004.

[46] H. Ramoser, J. Muller-Gerking, and G. Pfurtscheller, "Optimal spatial filtering of single trial EEG during imagined hand movement," Rehabil. Eng. IEEE Trans., vol. 8, no. 4, pp. 441-446, 2000.

[47] B. Blankertz, R. Tomioka, S. Lemm, M. Kawanabe, and K.-R Muller, "Optimizing spatial filters for robust EEG single-trial analysis," Signal Process. Mag. IEEE, vol. 25, no. 1, pp. 41-56, 2008.

[48] G. R. Müller-putz, R. Scherer, C. Brunner, R. Leeb, and G. Pfurtscheller, "Better than random? A closer look on BCI results," Int. Jouranl Bioelectromagn., vol. 10, no. 1, pp. 52-55, 2008.

[49] S. Ahn, K. Kim, and S. C. Jun, "Steady-State Somatosensory Evoked Potential for Brain-Computer Interface--Present and Future," Front. Hum. Neurosci., vol. 9, p. 716, 2015.

[50] S. Ahn, M. Ahn, H. Cho, and S. C. Jun, "Achieving a hybrid brain--computer interface with tactile selective attention and motor imagery," J. Neural Eng., vol. 11, no. 6, p. 66004, 2014.

[51] E. Houdayer, E. Labyt, F. Cassim, J. L. Bourriez, and P. Derambure, "Relationship between event-related beta synchronization and afferent inputs: Analysis of finger movement and peripheral nerve stimulations," Clin. Neurophysiol., vol. 117, no. 3, pp. 628-636, 2006.

[52] G. Pfurtscheller, C. Neuper, and others, "Motor imagery activates primary sensorimotor area in humans.," Neurosci. Lett., vol. 239, no. 2-3, p. 65, 1997.

[53] G. Pfurtscheller and F. H. da Silva, "Event-related EEG/MEG synchronization and desynchronization: basic principles," Clin. Neurophysiol., vol. 110, no. 11, pp. 1842-1857, 1999.

[54] L. Yao, X. Sheng, D. Zhang, N. Jiang, D. Farina, and X. Zhu, "A BCI System based on Somatosensory Attentional Orientation," IEEE Trans. Neural Syst. Rehabil. Eng., vol. 4320, no. c, pp. 1-1, 2016. 
